
\title{
R Research S Surare \\ Self-stabilization of levitating droplet clusters: A new idea and its experimental confirmation
}

\section{Leonid Dombrovsky ( $\sim$ ldombr@yandex.ru )}

Joint Institute for High Temperatures (RAS) https://orcid.org/0000-0002-6290-019X

\section{Alexander Fedorets}

University of Tyumen https://orcid.org/0000-0001-6595-3927

\section{Vladimir Levashov}

Institute of Mechanics of Moscow State University

Dmitry Shcherbakov

University of Tyumen

\section{Article}

Keywords:

Posted Date: February 7th, 2022

DOI: https://doi.org/10.21203/rs.3.rs-1273275/v1

License: (9) This work is licensed under a Creative Commons Attribution 4.0 International License. Read Full License 


\title{
Self-stabilization of levitating droplet clusters:
}

\section{A new idea and its experimental confirmation}

\author{
Alexander A. Fedorets, ${ }^{1}$ Dmitry V. Shcherbakov, ${ }^{1}$ \\ Vladimir Yu. Levashov, ${ }^{2}$ Leonid A. Dombrovsky ${ }^{1,3,4 *}$ \\ ${ }^{1}$ X-BIO Institute, University of Tyumen, 6 Volodarsky St., Tyumen 625003, Russia \\ ${ }^{2}$ Institute of Mechanics, Moscow State University, 1 Michurinsky Ave., Moscow 119192, Russia \\ ${ }^{3}$ Department of Chemical Engineering, Faculty of Engineering, Ariel University, Ariel 407000, Israel \\ ${ }^{4}$ Heat Transfer Department, Joint Institute for High Temperatures, 17A Krasnokazarmennaya St., Moscow \\ 111116, Russia
}

\begin{abstract}
A method of self-stabilization of a droplet cluster levitating over the locally heated water surface is proposed for the first time. The physical analysis of the problem shows that the desired result can be reached by dissolving a small amount of sodium chloride in a layer of water. This is explained by simultaneous action of two processes with opposite effects on evaporation. On the one hand, when water evaporates, a thin layer of increased salt concentration that prevents evaporation is formed at its surface. On the contrary, diffusion of salt in water decreases the surface concentration of salt. Different combinations of water heating intensity and average salt concentration are expected to result in the stable size of nearly identical levitating droplets, when there is a balance between the droplet evaporation and the condensation of vapor from the ascending vapor-air flow. The predicted phenomenon has been observed experimentally. In a series of laboratory experiments, a range of parameters has been obtained for which self-stabilization of the droplet cluster takes place. An analysis of the combined problem of heat transfer, water evaporation, and salt diffusion in water made it possible to obtain the minimum required value of the salt concentration, which is very close to that obtained experimentally.
\end{abstract}

\footnotetext{
* Corresponding author. E-mail: 1dombr@yandex.ru
} 


\section{Introduction}

It is known that small droplets are always suspended in humid air above heated water. These droplets condense from water vapor and positioned randomly over the water surface. In early paper [1], it was first discovered that, with local heating of water, droplets are self-arranged and form a flat ordered cluster, which levitates over the hot spot of the water surface. However, the droplet cluster exists for a very short time: as a result of the condensational growth of droplets, the cluster coalesces with the water layer. A method for cluster stabilization using the external infrared radiation, which heats the droplets and increases their evaporation has been developed by the authors [2]. Such a stabilization of droplets makes them suitable for chemical and biochemical studies in droplet micro-reactors [3]. Unfortunately, the technical characteristics of compact sources of the near-infrared radiation do not ensure their reliable long-term operation and the droplets are additionally heated, which may be undesirable in experiments with living microorganisms. The use of modulation of the heating power to prevent the condensational growth of droplets made it possible to reduce the droplet growth rate by about half [4], but this turned out to be insufficient. The foregoing motivated the analysis of a fundamentally different method for suppressing the growth of cluster droplets using self-regulation of the evaporation rate of the water layer. At the same time, it is also important to weaken the too strong temperature dependence of the evaporation rate of pure water. The use of an alternative technology developed by the authors for generating an initial cluster, when the droplets are supplied by an external source (see the corresponding section of the paper) made it possible, in particular, to work with a layer of water in which a non-evaporating substance is dissolved. In the present study, we consider sodium chloride (hereafter called simply "salt") as such a substance.

A physical analysis of the effects related with the addition of salt to the water layer is presented in the next section of the article. Theoretical estimates have shown that, in a certain range of parameters, a spontaneous stabilization of the droplet size is expected even at low average concentration of salt.

\section{Physical analysis of the problem}


First of all, let us compare the characteristic time of two processes: heating of a layer of water with a thickness $d$ and diffusion of salt in this water layer:

$$
t_{\text {heat }}=d^{2} / \kappa_{\mathrm{w}} \quad t_{\text {diff }}=d^{2} / D
$$

where $\kappa_{\mathrm{w}}$ is the thermal diffusivity of water (a small concentration of salt is neglected) and $D$ is the diffusivity of salt in water. In the temperature range of $40<T<80{ }^{\circ} \mathrm{C}, \kappa_{\mathrm{w}}$ increases slightly from $1.5 \times 10^{-7}$ to $1.7 \times 10^{-7} \mathrm{~m}^{2} / \mathrm{s}$, whereas $D$ increases with temperature from about $2.1 \times 10^{-9}$ to $4.4 \times 10^{-9} \mathrm{~m}^{2} / \mathrm{s}$. For $d=0.4 \mathrm{~mm}$, we obtain the values $t_{\text {heat }} \approx 1 \mathrm{~s}$ and $36<t_{\text {diff }}<76 \mathrm{~s}$. This means that the temperature profile in the water layer under the cluster can be established in one second, and it takes about one minute to establish the salt concentration profile.

Obviously, at the beginning of water evaporation, the salt concentration increases in a thin surface layer. At the same time, rather slow diffusion does not have time to counteract this local increase in salt concentration. Therefore, in the case of low-salinity water, the cluster droplets first grow until the evaporation rate decreases considerably. The latter follows the Raoult law, according to which the evaporation rate decreases with the increase in the surface salt concentration $c_{\text {surf }} \gg c_{\mathrm{av}}$, where $c_{\mathrm{av}}$ is the initial uniform concentration. After a time exceeding $t_{\text {diff }}$, one should expect a decrease in the size of water droplets in the cluster to a certain equilibrium value. At the same time, a steady-state profile of salt concentration over the thickness of the water layer can be achieved.

\section{Experimental results}

The scheme of the experiment is shown in Fig. 1. A description of the equipment and the experimental procedure is given in section Methods. The experiments were carried out at three values of the average mass concentration of salt in the water layer: $c_{\mathrm{av}}=0.2 \%, 0.3 \%$, and $0.4 \%$, but the first of these values appeared to be insufficient to obtain the self-stabilized cluster of levitating water droplets.

Here we show only some results of the laboratory experiments. At the water surface temperature of $T_{\text {surf }}=60 \mp 0.5^{\circ} \mathrm{C}$ and two values of the salt concentration of $c_{\mathrm{av}}=0.3 \%$ and $0.4 \%$, small clusters of 11 and 13 droplets are formed, respectively (see Methods). Photographs of these clusters at different 
points in time are shown in Fig. 2. The change in the radius of water droplets over time is clearer in

Fig. 3. Obviously, these results confirm the above theoretical predictions. As one might expect, the dependences $a(t)$ at different values of $c_{\mathrm{av}}$ are similar to each other, but at a higher salt concentration in the water layer, the cluster droplets are smaller and their initial growth stops earlier, after which the droplet radius decreases to the equilibrium value $a_{\mathrm{eq}}$. It is interesting that the results for two values of salt concentration practically coincide with each other (except for the regime close to equilibrium) when the coordinates $(t, a)$ are replaced by $\left(c_{\mathrm{av}} t, \sqrt{c_{\mathrm{av}}} a\right)$, which looks to be expected according to the usual criteria for diffusion-like processes when we are dealing with relationships like $t / d^{2}$ (as in conductive and diffusion Fourier numbers).

It turns out that an increase in water temperature is favorable for the self-stabilization of the cluster. As shown in Fig. 4, at $T_{\max }>74^{\circ} \mathrm{C}$ this effect is observed even at $c_{\mathrm{av}}=0.2 \%$. For any values of $c_{\mathrm{av}}$, the equilibrium radius of droplets $a_{\mathrm{eq}}$ increases almost linearly with increasing the water temperature.

\section{Computational results}

To obtain the quantitative results for the evaporation problem in the case of a layer of saline water, one should solve coupled equations of heat conduction and salt diffusion accompanied by the evaporation model. The axisymmetric heat conduction problem should take into account both the axial and radial heat transfer in the working section of the laboratory set-up. The mathematical formulation and algorithm for solving the thermal problem do not differ from those used in [6], but the new calculations were performed for other geometric dimensions and took into account the cooling of the modified metal cuvette. A typical temperature field in the central part of the water layer is shown in Fig. 5. This temperature field seems to be rather complex, but the steady-state profile of water temperature under the cluster (along the vertical axis of the computational region) is obviously linear:

$$
T(z)=T_{\max }-\left(T_{\max }-T_{\text {surf }}\right) z / d \quad 0<z<d
$$

where $z$ is the coordinate measured from the lower boundary of the water layer. 
At the maintained thickness of the water layer, the transient diffusion equation for the current mass concentration of salt, $c(t, z)$, and the initial and boundary conditions can be written as follows:

$$
\begin{aligned}
& \frac{\partial c}{\partial t}=\frac{\partial}{\partial z}\left(D(z) \frac{\partial c}{\partial z}\right)-\frac{u_{\text {surf }}}{d}, \quad t>0, \\
& t=0, \quad c=c_{\mathrm{av}} ; \quad z=0, \quad \frac{\partial c}{\partial z}=0 ; \quad z=d, \quad D(d) \frac{\partial c}{\partial z}=u_{\text {surf }}
\end{aligned}
$$

where $u_{\text {surf }}=\dot{m} / \rho_{\mathrm{w}}, \dot{m}\left(T_{\text {surf }}, c_{\text {surf }}\right)$ is the mass rate of water evaporation, and $\rho_{\mathrm{w}}$ is the density of water. The last term in the right-hand side of Eq. (3a) corresponds to the addition of water to maintain a constant layer thickness in the experiment (see section Methods). The coordinate dependence of $D$ is related with its dependence on temperature:

$$
D(T)=D_{0}(T-273) /\left(T_{0}-273\right)
$$

where $T$ is measured in Kelvin, $T_{0}=333 \mathrm{~K}$, and $D_{0}=3.3 \times 10^{-9} \mathrm{~m}^{2} / \mathrm{s}$. Equation (4) is a linear approximation of the experimental data [5].

Strictly speaking, a complete model of evaporation should include consideration of a thin Knudsen layer at the water surface [8]. However, in the present paper we limit ourselves by the diffusion approach taking into account the Stefan flow [9]:

$$
\dot{m}=\frac{D_{\text {gas }}\left(T_{\text {surf }}\right) p}{H_{\mathrm{e}} R_{\text {mix }} T_{\text {surf }}} \ln \frac{1-\varphi_{\mathrm{e}} \bar{p}_{\text {sat }} \bar{\mu}_{\mathrm{w}}}{1-\xi_{\mathrm{w}} \bar{p}_{\text {sat }} \bar{\mu}_{\mathrm{w}}}, \quad \bar{p}_{\text {sat }}=\frac{p_{\text {sat }}\left(T_{\text {surf }}\right)}{p}, \quad \bar{\mu}_{\mathrm{w}}=\frac{\mu_{\mathrm{w}}}{\mu_{\text {mix }}}
$$

where $D_{\text {gas }}$ is the diffusion coefficient of water vapor in air, $p$ is the atmospheric pressure, $p_{\text {sat }}\left(T_{\text {surf }}\right)$ is the saturation pressure of water vapor at temperature $T_{\text {surf }}, \mu_{\mathrm{w}}$ and $\mu_{\text {mix }} \approx \mu_{\text {air }}$ are molar masses of water vapor and the mixture of air with the vapor, $R_{\text {mix }} \approx R_{\text {air }}=R / \mu_{\text {air }}(R$ is the universal gas constant), $H_{\mathrm{e}}$ is the distance from the water surface at which the relative humidity of air is equal to $\varphi_{\mathrm{e}}$, and $\xi_{\mathrm{w}}$ is the local mole fraction of water in salt solution, which can be expressed through the mass concentration of salt at the surface of the water layer:

$$
\xi_{\mathrm{w}}=1 /\left(1+\tilde{\mu}_{\mathrm{w}} c_{\text {surf }} /\left(1-c_{\text {surf }}\right)\right) \quad \tilde{\mu}_{\mathrm{w}}=\mu_{\mathrm{w}} / \mu_{\text {salt }}
$$

where $\mu_{\text {salt }}$ is the molar mass of $\mathrm{NaCl}$. Note that the coefficient $\xi_{\mathrm{w}}$ in Eq. (5) corresponds to Raoult's law for the evaporation of a mixture of liquids or solutions. We use an approximate value of $H_{\mathrm{e}}$ obtained from a comparison of the diffusion approach with the exact kinetic model of evaporation [8].

The following approximation was used for the diffusion coefficient of water vapor [10]: 


$$
D_{\text {gas }}(T)=-2.775 \times 10^{-6}+4.479 \times 10^{-8} T+1.656 \times 10^{-10} T^{2}
$$

where $D_{\text {gas }}$ is measured in $\mathrm{m}^{2} / \mathrm{s}$. According to [11] the saturation pressure (in $\mathrm{Pa}$ ) is approximated as:

$$
\lg p_{\text {sat }}=9.6543-1435.264 /(T-64.848)
$$

In both Eqs. (7) and (8), the temperature is measured in Kelvin.

First of all, it is interesting to look at the calculated effect of the surface concentration of the dissolved salt on the mass rate of water evaporation. This effect is clear in Fig. 6, where the variation of the relative value $\dot{m}_{\text {rel }}=\dot{m}\left(T_{\text {surf }}, c_{\text {surf }}\right) / \dot{m}\left(T_{\text {surf }}, 0\right)$ with $c_{\text {surf }}$ at various temperatures is presented. The value of $\dot{m}_{\text {rel }}$ decreases almost linearly with the concentration of salt at $c_{\text {surf }}<10 \%$, and this effect is weakly sensitive to the water temperature in the range of $60<T_{\text {surf }}<80{ }^{\circ} \mathrm{C}$. Therefore, our subsequent analysis for the value of $T_{\text {surf }}=60{ }^{\circ} \mathrm{C}$ is representative.

It is obvious in Fig. 3 that a variation of the concentration of salt near the surface of the water layer is negligible in the initial period of $t<30 \mathrm{~s}$ when there is no considerable effect of salt on the evaporation rate of water. On the other hand, there is a subsequent variation in the radius of the levitating droplets up to the equilibrium value $a_{\mathrm{eq}}$. Therefore, it seems reasonable not to solve the transient diffusion problem $(3 a, b)$ but focus on the steady-state solution which can be obtained analytically. The steady-state problem for the profile of the salt concentration can be written as follows:

$$
\begin{array}{cl}
\frac{d}{d \bar{z}}\left(D(\bar{z}) \frac{d c}{d \bar{z}}\right)=S, & \left(\frac{d c}{d \bar{z}}\right)_{\bar{z}=0}=0, \quad D(1)\left(\frac{d c}{d \bar{z}}\right)_{\bar{z}=1}=S \\
& \int_{0}^{1} c(\bar{z}) \mathrm{d} \bar{z}=c_{\mathrm{av}}
\end{array}
$$

where $\bar{z}=z / d, S=u_{\text {surf, } 0} d$, and the value of $u_{\text {surf, } 0}$ corresponds to the steady-state regime. Note that the temperature difference across the water layer is only about thirty degrees and the salt diffusion is limited by the diffusion coefficient near the water surface. Due to this, the diffusion coefficient can be considered constant: $D(\bar{z})=D(1)=D_{\text {surf }}$. In this case, the concentration profile is parabolic:

$$
c(\bar{z})=c_{\mathrm{av}}+\frac{S}{2 D_{\text {surf }}}\left(\bar{z}^{2}-\frac{1}{3}\right)
$$


To calculate this concentration profile, it is necessary to know the parameter $S$, which is determined by the value of the equilibrium salt concentration on the water surface $c_{\text {surf }}$. This can be done by solving the following transcendental equation:

$$
\dot{m}\left(T_{\text {surf }}, c_{\text {surf }}\right) d / \rho_{\mathrm{w}}=3 D_{\text {surf }} \times\left(c_{\text {surf }}-c_{\text {av }}\right)
$$

in the left and right parts of which are different expressions for $S$ (see Eq. (10) at $\bar{z}=1$ ). The resulting solution for the profiles of the salt concentration is presented in Fig. 7.

It is important that the above analytical solution for the steady-state profile of the salt concentration cannot be obtained when Eq. (10) gives a negative value of $c(0)$. This leads to the following critical condition for the self-stabilization of the droplet cluster:

$$
c_{\mathrm{av}}>c_{\mathrm{av}}^{*}\left(T_{\text {surf }}\right)=\frac{\dot{m}\left(T_{\text {surf }}\right) d}{6 \rho_{\mathrm{w}} D_{\text {surf }}}
$$

We have omitted here a weak parameter $c_{\text {surf }}$ in the expression for $\dot{m}$. The calculations using the approximate Eq. (12) at $T_{\text {surf }}=60{ }^{\circ} \mathrm{C}$ yields the critical value of $c_{\mathrm{av}}^{*}=0.17 \%$. This agrees well with the mentioned experimental results at $c_{\mathrm{av}}=0.2 \%$, when a monotonic growth of droplets with subsequent coalescence of the cluster with the water layer was observed.

Following traditional practice, a dimensionless self-stabilization parameter can be proposed:

$$
S_{D}=\frac{S}{c_{\mathrm{av}} D_{\mathrm{surf}}}
$$

The self-stabilization of the droplet cluster takes place at $S_{D}<6$. This, in particular, means that the stabilization of a droplet cluster over a layer of salt water is possible only at a not too low salt concentration. It is interesting to apply the introduced criterion to fairly complete experimental results obtained at $c_{\mathrm{av}}=0.3 \%$ (Fig. 4). With an increase in the water surface temperature from 60 to $78{ }^{\circ} \mathrm{C}$, the intensity of water evaporation, which can be characterized by the value of $S$, increases by about 2.6 times. It would seem that this may lead to a violation of the cluster stabilization condition. But with the above increase in water temperature, the diffusion coefficient of salt in water $D_{\text {surf }}$ also increases by a factor of 1.3. As a result, even at $c_{\mathrm{av}}=0.3 \%$, the calculated parameter $S_{D}$ changes from 3.2 at $T_{\text {surf }}=$ $60{ }^{\circ} \mathrm{C}$ to 6.3 at $T_{\text {surf }}=78{ }^{\circ} \mathrm{C}$ and the approximate condition $S_{D}<6$ is still applicable. 


\section{Conclusions}

The laboratory experiments confirmed that the developed method of self-stabilization of a levitating droplet cluster is really effective when the mass concentration of sodium chloride in water is greater than $0.2 \%$. It was shown that the size of nearly identical droplets in the equilibrium cluster decreases inversely proportional to the average concentration of salt in water. The theoretical analysis of the minimum required salt concentration in the water layer agrees well with the experimental finding. Moreover, a dimensionless criterion is proposed for estimating the conditions for self-stabilization of the droplet cluster. The developed method and the suggested theoretical model open up new possibilities for studying chemical and biochemical processes in small droplets, which can be used as natural micro-reactors. The results obtained can also be interesting for studies of processes in the surface layer of the ocean and in the analysis of the remote sensing results.

\section{Methods}

The schematic of the experiment is shown in Fig. 1a, where 1 - the droplet cluster, 2 - the layer of water with small concentrations of a surfactant and salt, 3 - the sitall substrate, 4 - the cuvette with a sealed channel connected to the external circuit of a cryothermostat, 5 - the laser beam, $6-$ microscope and thermal imager lens. A $0.4 \mathrm{~mm}$ thick sitall substrate is glued to the metal bottom of the cuvette. The cryothermostat CC 805 (Huber, Germany) with the coolant temperature of $10 \pm 1$ ${ }^{\circ} \mathrm{C}$ allows stabilizing the temperature of water layer. The water layer is heated by a laser beam (MRL-III-660D-1W, manufacturer - CNI, China) aimed at the lower blackened surface of the substrate. The radial temperature profile of the water layer surface (Fig. 1b) was recorded with a CG640 thermal imager (COX, Korea). Video recording of the cluster image was carried out using an AXIO Zoom.V16 stereomicroscope (Zeiss, Germany) equipped with a camera Andor iXon Ultra 888 (Oxford Instruments plc, UK). The thickness of the water layer is equal to $0.4 \mathrm{~mm}$. It was controlled by a laser confocal sensor IFC2451 (Micro-Epsilon, USA) and maintained with an accuracy of $\pm 2 \mu \mathrm{m}$. The experiments were carried out with salt solutions (impurity content less than 
$0.1 \%$ ) in distilled water. The mass concentration of salt in water was varied. The solution always contained a surfactant (sodium lauryl sulfate) with a concentration of $0.02 \mathrm{~g} / \mathrm{l}$, which is necessary to suppress the thermocapillary flow in the water layer [7]. The water surface temperature under the cluster was monitored with a CTL-CF1-C3 pyrometric sensor (Micro-Epsilon, USA). The spectral range of the sensor sensitivity was from 8 to $14 \mu \mathrm{m}$, and the resulting error is equal to $\mp 0.1{ }^{\circ} \mathrm{C}$.

A small cluster with the number of droplets from 10 to 20 was "printed" from distilled water by injection of microdroplets with a piezoelectric dispenser (MicroFab Technologies, Inc., USA). The use of small clusters is explained by the fact that such clusters are located near the maximum temperature of the surface of the water layer (Fig. 1b), which provides almost the same external conditions for all droplets in the cluster.

After that, a pause of about $300 \mathrm{~s}$ was maintained, which was necessary to stabilize the droplet size, and the cluster was recorded. Then, the laser heating power was increased by $20 \mathrm{~mW}$. The equilibrium droplet radius, $a_{\mathrm{eq}}$, was measured for several values of the laser heating power. Each $a_{\text {eq }}$ value was calculated over 50 consecutive frames as the average radius of all droplets in the cluster.

Acknowledgements: The authors are grateful to the Russian Science Foundation (project 19-1900076) for the financial support of the work. We also thank our colleagues Alexei Kryukov and Anatoly Levin for their interest and participation in the discussion of the work.

Author contributions: Conceptualization, A.A.F. and L.A.D; A.A.F. and D.V.S. designed the experimental set-up and performed the experiments; L.A.D. developed the physical model; L.A.D. and V.Y.L. performed the calculations, L.A.D. wrote the draft manuscript; All authors have read and agreed to the final version of the manuscript.

Competing interests: The authors declare no conflict of interest.

\section{References}

1. Fedorets, A.A. Droplet cluster, JETP Lett. 79, 372-374 (2004).

2. Dombrovsky, L.A., Fedorets, A.A., Medvedev, D.N. The use of infrared irradiation to stabilize levitating clusters of water droplets, Infrared Phys. Techn. 75, 124-132 (2016). 
3. Fedorets, A.A., Bormashenko, E., Dombrovsky, L.A., Nosonovsky M. Droplet clusters: Natureinspired biological reactors and aerosols, Philos. Trans. R. Soc. A 377, 20190121 (2019).

4. Fedorets, A.A., Aktaev, N.E., Dombrovsky, L.A. Suppression of the condensational growth of droplets of a levitating cluster using the modulation of the laser heating power, Int. J. Heat Mass Transfer 127A, 660-664 (2018).

5. Fell, C.J.D., Hutchison, H.P. Diffusion coefficients for sodium and potassium chlorides in water at elevated temperatures, J. Chem. Eng. Data 16, 427-429 (1971).

6. Fedorets, A.A., Dombrovsky, L.A. Generation of levitating droplet clusters above the locally heated water surface: A thermal analysis of modified installation, Int. J. Heat Mass Transfer 104, 1268-1274 (2017).

7. Fedorets, A.A., Shcherbakov, D.V., Dombrovsky, L.A., Bormashenko, E., Nosonovsky, M. Impact of surfactants on the formation and properties of droplet clusters, Langmuir 36, 1115411160 (2020).

8. Levashov, V.Yu., Kryukov, A.P., Numerical simulation of water droplet evaporation into vapor-gas medium, Colloid J. 79, 647-653 (2017).

9. Sobac, B., Talbot, P., Haut, B., Rednikov, A., Colinet, P. A comprehensive analysis of the evaporation of a liquid spherical drop, J. Colloid Interface Sci. 438, 306-317 (2015).

10. Nellis, G., Klein, S. Heat Transfer. New York: Cambridge University Press (2009).

11. Stull, D.R. Vapor pressure of pure substances. Organic and inorganic compounds, Ind. Eng. Chem. 39, 517-540 (1947).
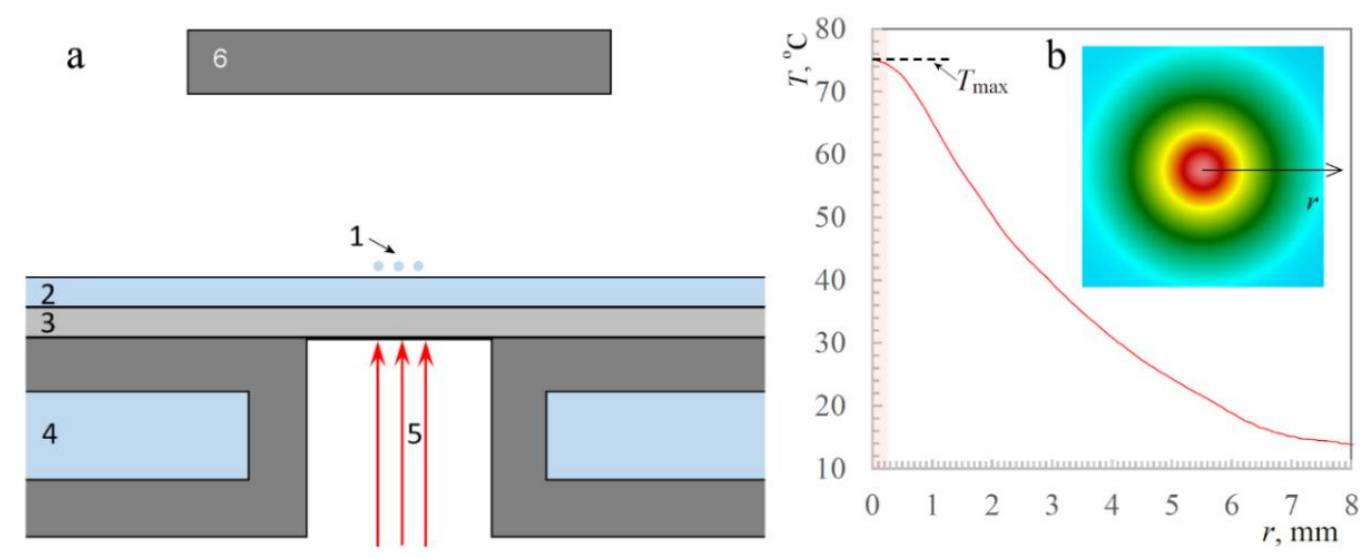

Fig. 1. (a) Schematics of experimental set-up; (b) Typical temperature profile at the water surface. The pink stripe shows the area of cluster localization. 


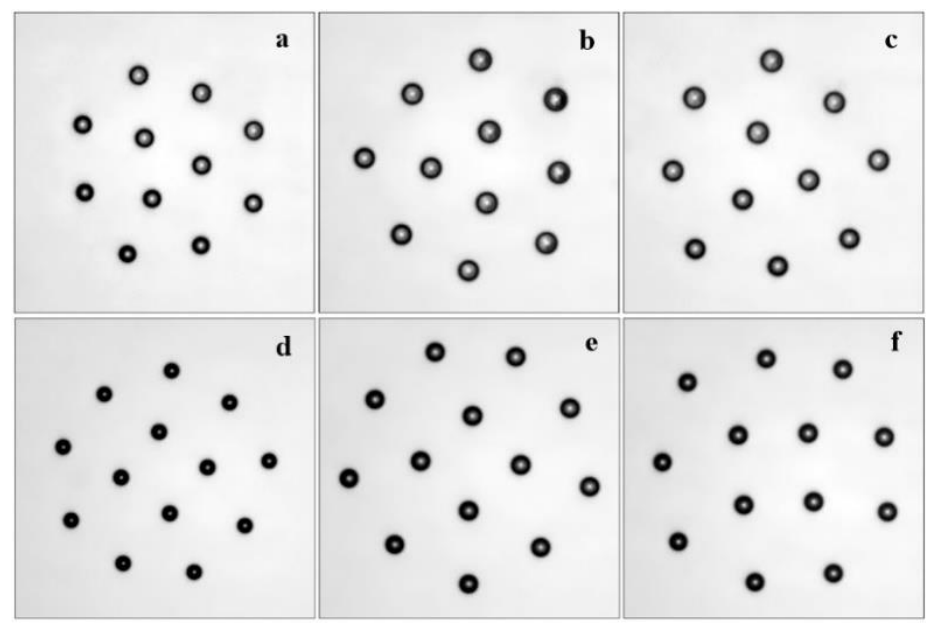

Fig. 2. Images of small water clusters for $c_{\mathrm{av}}=0.3 \%(\mathrm{a}, \mathrm{b}, \mathrm{c})$ and $0.4 \%(\mathrm{~d}, \mathrm{e}, \mathrm{f})$ : $\mathrm{a}, \mathrm{d}-t=0 ; \mathrm{b}, \mathrm{e}-90 \mathrm{~s} ; \mathrm{c}, \mathrm{f}-290 \mathrm{~s}$.

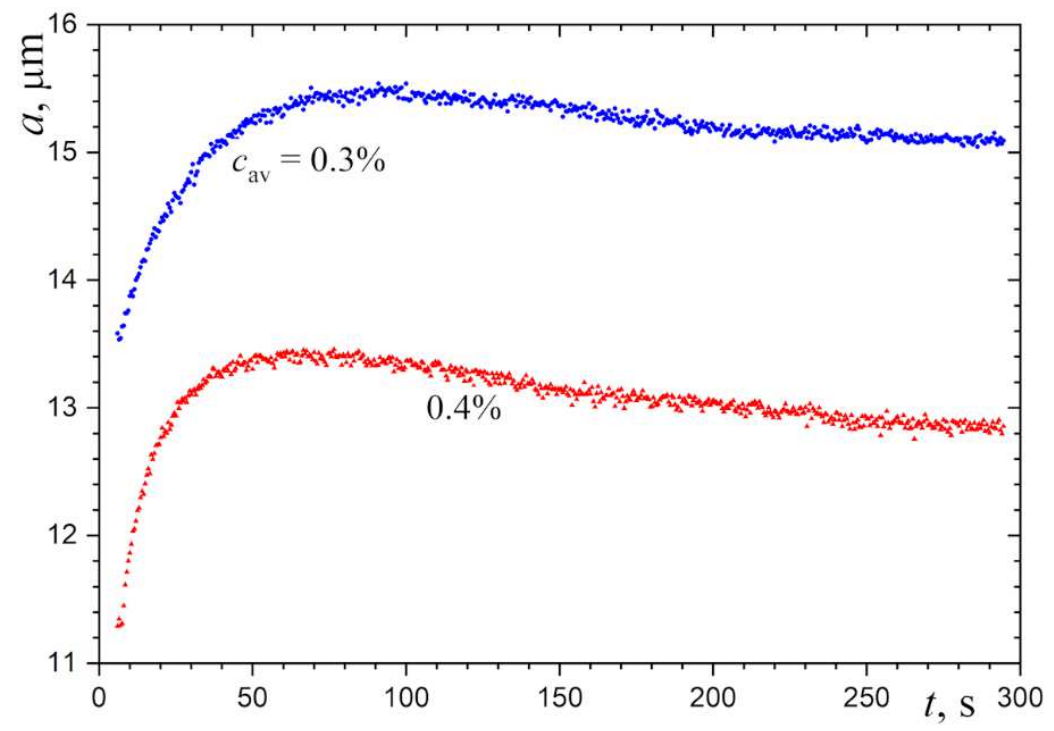

Fig. 3. Time variation of radius of water droplets in two clusters.

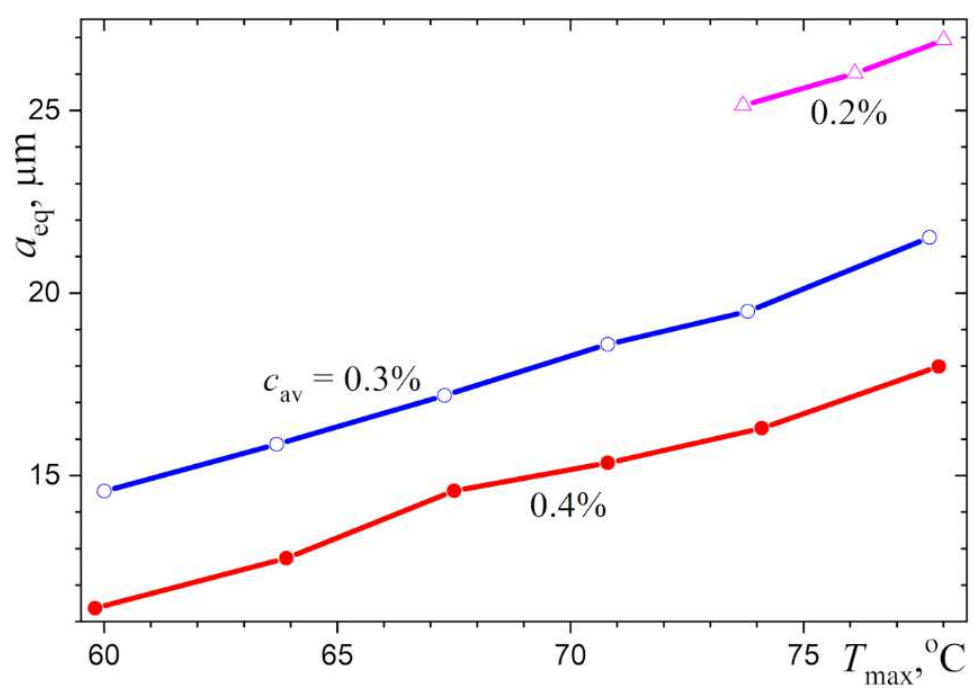

Fig. 4. Equilibrium radius of water droplets in two clusters. 


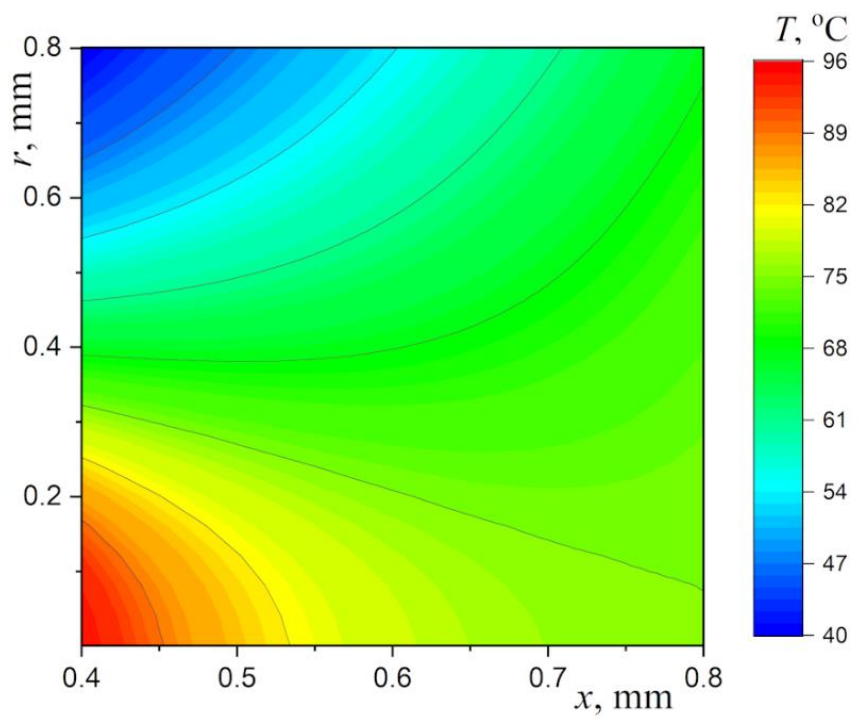

Fig. 5. Typical temperature field in the central part of the water layer

(the open water surface is on the right; the substrate is on the left);

The axial coordinate $x$ is measured from the bottom of the sitall substrate.

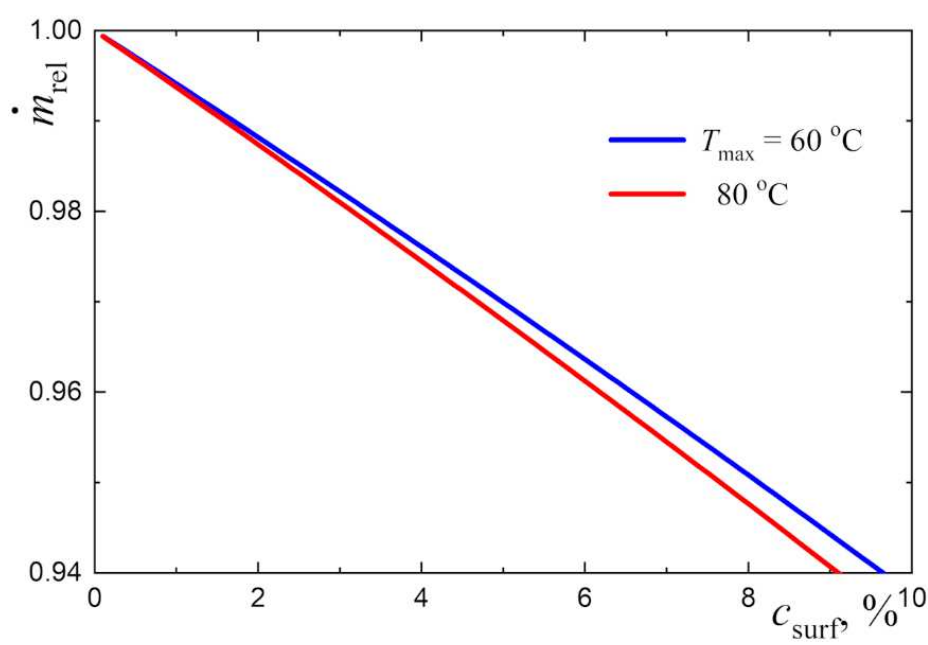

Fig. 6. Effect of the surface concentration of salt on the evaporation rate of water.

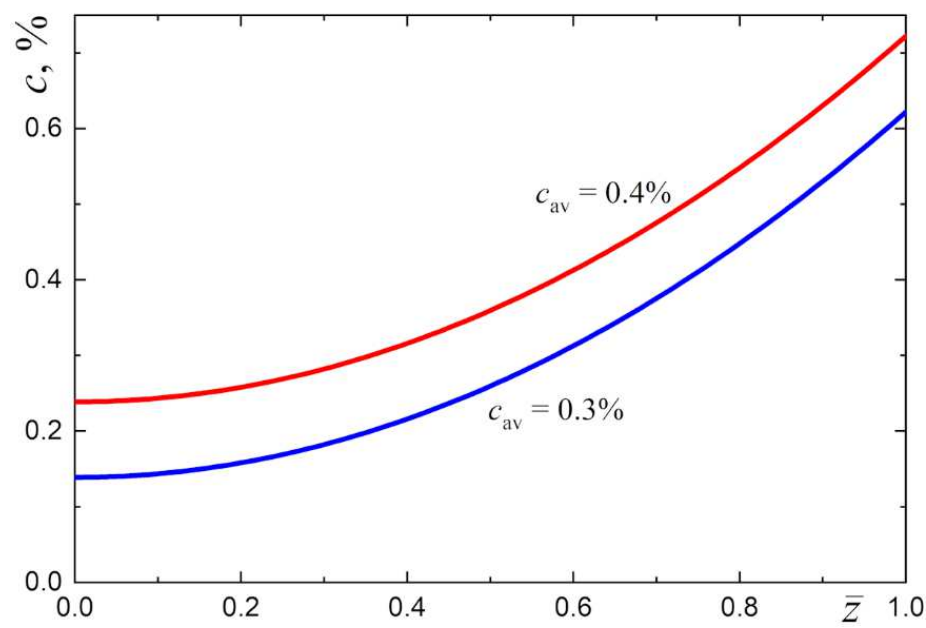

Fig. 7. Steady-state profiles of salt concentration in the water layer under the equilibrium cluster of levitating water droplets. 